4. Dijkstra E.W. The Structure of the "THE"-Multiprogramming System. In Communications of the ACM, 11 (5), May 1968 (pp. 341-346).

5. Liskov B.H. A Design Methodology for Reliable Software Systems. In Proc. of AFIPS'72 (Fall, part I), 1972 (pp. 191-199).

6. Basic. A Manual for BASIC, the elementary algebraic language designed for use with the Darthmouth Time Sharing System. Dartmouth College, 1964. Available at: http://www.bitsavers.org/pdf/ dartmouth/BASIC_Oct64.pdf (дата обращения: 04.09.2021).

7. Bogardus Cortez, M. History of Basic Computer Programming Lan-guages: C Language, BASIC and Beyond. In EdTech magazine, June 2017. Available at: https://edtechmagazine.com/higher/article/2017/06/ historyprogramming-languages-c-language-basic-and-beyond (дата обращения: 10.09.2021).

8. Niklaus Wirth. The Programming Language Pascal. In Acta Informatica, 1, 1971 (pp. 35-63).

9. Chapman, M.R. In Search of Stupidity: Over 20 Years of High-Tech Marketing Disasters, 2nd ed. Apress, 2006.

10. Gibson, S. Borland and Microsoft Enter the Object-Oriented Pascal Ring. In Infoworld, 11 (9), 1989 (p. 28 ).

11. Srinath K.R. Python - The Fastest Growing Programming Language. In International Research Journal of Engineering and Technology (IRJET), 4 (12), 2017 (pp. 354-357).

12. Cass, S. The Top Programming Languages 2018. Python stays on top, and Assembly enters the Top Ten. In IEEE Spectrum, July 2018. Available at: https://spectrum.ieee.org/at-work/innovation/the-2018-top-programminglanguages (дата обращения: 15.09.2021).

13. Ritchie, D. The Development of the C language. In HOPL-II The 2nd ACM SIGPLAN conference on History of Programming Languages, Cambridge, Massachusetts, USA, 1993 (pp. 201-208).

14. https://pascalabcnet.github.io/mydoc_for_EGE.html (дата обращения 22.09.2021).

15. Осипов A.B. РascalABC.NET: Введение в современное программи-рование. - Ростов-на-Дону, 2019- 572 с.

16. Осипов A.B. РascalABC.NET: выбор школьника. Часть 3. /А.В. Осипов. - Ростов-на-Дону, 2020 - 146 с.

17. Лутц, Марк. Изучаем Pуthon, том 1, 5-е изд.: Пер. с англ. - СПб.: ООО “Диалектика”, 2019. - 832 с.

18. Лутц, Марк. Изучаем Python, том 2, 5-е изд.: Пер. с англ. - СПб.: ООО “Диалектика”, 2020. - 720 с.

19. https://habr.com/ru/post/543346/ (дата обращения 20.05.2021).

20. http://www.ptaskbook.com/ru/ (дата обращения 28.09.2021).

\title{
Смирнов С.В. \\ Проектирование тематического слоя электронной карты при помощи графа качественных признаков объектов
}

ИПУ РАН

(Россия, Москва)

doi: 10.18411/trnio-10-2021-46

\section{Аннотация}

В докладе описано проектирование тематического слоя электронной карты специализированной геоинформационной системы (ГИС) при помощи мозаичной иерархической модели изобразительных средств. Рассмотрен граф понятийно содержательных признаков. Рассмотрена схема графа формирования изобразительных средств для проектирования знаков. Обосновано применение способа квадратомического дерева для ГИС.

Ключевые слова: геоинформационной системы (ГИС), граф, модель, тематический слой, электронная карта, картография, квадратомическое дерево, иерархическая структура, графическая информация.

\section{Abstract}

The report describes the design of the thematic layer of the electronic map of a specialized geoinformation system (GIS) using a mosaic hierarchical model of visual means. The graph of conceptual and substantive features is considered. The scheme of the graph of the formation of visual means for the design of signs is considered. The application of the quadratic tree method for GIS is justified.

Keywords: geographic information system (GIS), graph, model, thematic layer, electronic map, cartography, quadratic tree, hierarchical structure, graphic information. 


\section{Введение}

При разработке геоинформационной системы важное место занимает проектирование её оформления. От правильного оформления карты зависит её читаемость и наглядность, а также эстетические достоинства. Проектирование карты - сложный творческий процесс, требующий больших знаний и опыта, постоянных поисков и экспериментов. На практике приходится учитывать многие требования, предъявляемые к оформлению любой электронной карты, знать специфику оформления разных типов геоинформационных систем (ГИС), достоинства и недостатки изобразительных средств. Зачастую через внешний вид спроектированной системы определяется ценность её содержания. Необходимо заметить, что наглядность свойство психологическое. Однако, чтобы до конца понять содержание физической системы (тематический слой электронной карты), которая моделируется необходимо стремиться к максимальной наглядности. Познание мира есть процесс его последовательного осмысления, а не просто его описания [1, с. 344].

Разработка необходимой модели данных тематического слоя - одна из важнейших задач при построении электронной карты ГИС. Основные проблемы обработки, эффективности, хранения и удобства использования остаются в связи с наличием векторных и растровых моделей данных.

Решение этих проблем достигается созданием мозаичных иерархических моделей. Мозаичность моделей, получается, из-за разделения плоскости данных на многоугольники, которые могут быть квадратами, треугольниками и т.п.

\section{1. Передача пространственных форм графо-аналитической модели}

Немного терминов и определений. Многие объекты, возникающие в жизни человека, могут быть смоделированы (представлены в памяти компьютера) при помощи графов. Например, транспортные схемы (схема метрополитена и т. д.) изображают в виде станций, соединенных линиями. В терминах графов станции называются вершинами графа, а линии рёбра.

Графом называется конечное множество вершин и множество ребер. Каждому ребру сопоставлены две вершины - концы ребра [2].

Бывают различные варианты определения графа. В этом определении концы у каждого ребра - равноправны. В этом случае нет разницы где начало, а где - конец у ребра. Однако, в транспортных сетях бывают случаи одностороннего движения по ребру, тогда говорят об ориентированном графе - это когда у ребер которого одна вершина считается начальной, а другая - конечной.

Рёбра называются кратными, если они соединяют одну и ту же пару вершин (а в случае ориентированного графа - если у них совпадают начала и концы). Ребро называется петлей, если у него совпадают начало и конец. Во многих задачах кратные рёбра и петли не представляют интереса, поэтому могут рассматриваться только графы без петель и кратных ребер. Такие графы называют простыми.

Степенью вершины в неориентированном графе называется число инцидентных данной вершине ребер. Довольно очевидно, что сумма степеней всех вершин равна удвоенному числу ребер в графе. Отсюда можно посчитать максимальное число ребер в простом графе - если у графа вершин, то степень каждой из них равна, а, значит, число ребер есть. Граф, в котором любые две вершины соединены одним ребром, называется полным графом.

Также легко заметить следующий факт - в любом графе число вершин нечетной степени - четно. Этот факт называется «леммой о рукопожатиях» - положение теории графов, согласно которому любой конечный неориентированный граф имеет чётное число вершин нечётных степеней. Лемма берёт название от популярной аналогии: в группе людей, некоторые из которых пожимают друг другу руки, чётное число людей поприветствовало таким образом нечётное число коллег [3].

Проектирование графо-аналитической модели тематического слоя электронной карты для ГИС состоит из этапов разработки знаков передачи пространственных форм 
определённых качественных и количественных характеристик объектов, а также установления изобразительных средств применительно к содержанию, масштабу и назначению карт.

Проектирование знаков для передачи пространственных форм определённых качественных и количественных характеристик объектов целесообразней всего проводить при помощи машинно-ориентированного формализованного картографического языка (МФКЯ). Для этого применяют теорию графов. Применении теории графов в разработке карт впервые было выдвинуто и развито российским картографом А.С. Васмутом в труде [4].

Граф качественных признаков объектов (точечных, линейных и площадных) имеет общий вид иерархического дерева, построенного при помощи теории графов (рис. 1). Граф $G$ включает подграфы понятийных уровней. Каждый уровень определяется признаком общности и числом разнообразия этого признака: количеством вершин подграфа.

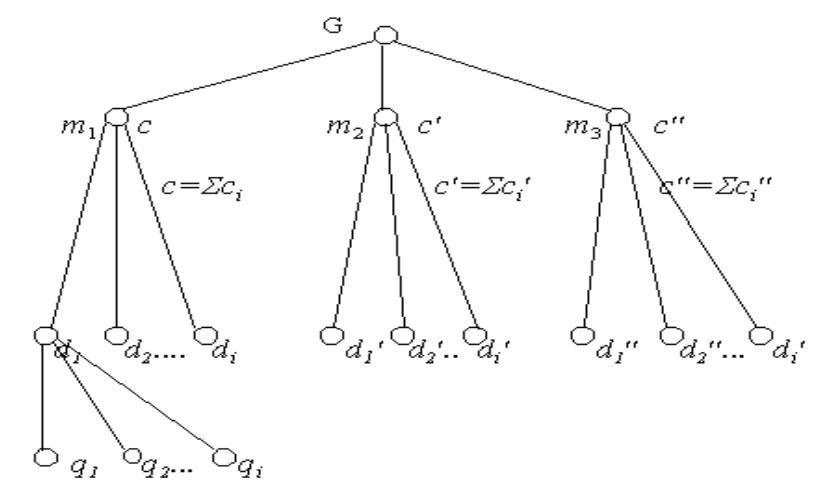

Рисунок 1. Граф понятийно-содержательных признаков

Как показано на рисунке граф $G$ на первом уровне включает несколько подграфов $c_{i}$. $\mathrm{B}$ некоторый подграф $c$ включены подграфы $d_{1}, d_{2}, \ldots, d_{i}$. Число вершин подграфа $c$ равно сумме вершин подграфов $d_{i}$. При последующем разделении граф $d_{i}$ имеет следующие уровни, в которые входят подграфы $q_{1}, q_{2}, \ldots, q_{i}$. Причём $d_{i}$ определяет их общность, а признаки $q_{i}{ }^{-}$ различия. Исходя из этого составляется граф изобразительных средств [5, с.19].

Граф изобразительных средств будет иметь вид иерархического дерева, где в вершинах будут находиться параметры знаков, которые определяют признаки общности и различия уровней характеристик объекта. В виду того, что объектам картографирования свойственна общность, которая выражается в единстве структурной организации системообразующих признаков, виды графов качественных признаков для разных объектов будут отличаться по числу уровней и элементам разнообразия [6, с. 63].

\section{2. Применение способа квадратомического дерева для ГИС}

У всех графов изобразительных средств для одинаковых (общих) уровней может быть принято единое средство изображения объекта. Средства изображения опираются в первую очередь на подходы, которые позволяют экономить объёмы, требуемые для хранения растровых данных, и способствуют сокращению времени доступа к элементам описания пространственных объектов. Одним из таких подходов является способ квадратомического дерева.

Применение модели квадратомического дерева, как объекта разработок для графических информационных систем представлен в работе [7, с. 184].

Иерархическая структура данных, известная как квадратомическое дерево, используется для накопления и хранения графической информации. В этой структуре двухмерная геометрическая область рекурсивно подразделяется на квадраты, что определило название данной модели.

Технология построения квадратомического дерева основана на рекурсивном разделении квадрата на квадранты и подквадранты до тех пор, пока все подквадранты не 
станут однородными по отношению к значению изображения (цвета, смысловая нагрузка) или пока не будет достигнут предопределенный заранее наименьший уровень разрешения.

Преимущество такой структуры состоит в том, что регулярное разделение обеспечивает накопление, восстановление и обработку данных простым и эффективным способом. Простота проистекает из геометрической регулярности разбиения, а эффективность достигается за счёт хранения только узлов с данными, которые представляют интерес [8].

Поскольку квадратомическое дерево известно как пространственно-рациональный способ представления сгруппированных однородных сопряженных изображений, их преимущество над векторной репрезентацией многих (но не всех) пространственных алгоритмов относительно затрат машинного времени весьма существенно.

Первоначально большинство приложений моделей квадратомических деревьев было сделано для обработки изображений. Из этой области данная модель была перенесена в ГИС.

В квадратомических структурах, которые разбивают изображение на вложенные ячейки, достигаются именно, те требования, которые необходимы для удобства формирования изобразительных средств. Этот подход применяется для представления растровых и векторных структур графических данных. Применение именно этой модели при организации данных для проектирования ГИС характерны следующим:

- $\quad$ больший квадрант становится узлом более высокого иерархического уровня квадратомического дерева, а меньшие квадранты на более низких уровнях;

- $\quad$ расчёты оптимальных площадей размещения знаков, центроидные определения, ускоренное распознавание образов;

- преобразование расстояний с целью максимально точной передачи метрических данных и др.

Существуют примеры, когда способ квадратомического дерева применялся для представления тематических данных с целью моделирования содержания некоторых видов карт (например: синтетические карты).

\section{Заключение}

Преимущества модели квадратомического дерева заключаются в пространственных связях закодированых в самой модели и осуществлении быстрого поиска, при увеличении однородности данных, а также их формирование в более компактный вариант [9, с.185].

Наряду с преимуществами такой модели представления данных встречаются, конечно, и недостатки. В обобщённом варианте недостатки выглядят так: процесс проектирования квадратомических деревьев трудоёмкий, при каком-либо изменении в плоскости данных требуется пересчёт всего дерева, а также эти модели не всегда эффективны при хранении спутниковых данных и сведений о цифровании территорий.

1. Акимов О.Е. Дискретная математика: логика, группы, графы. - 2-е изд., доп. - М.: Лаб. базовых знаний, 2001. $-376 \mathrm{c}$.

2. Теория графов, 2021 URL: https://foxford.ru/wiki/informatika/teoriya-grafov

3. Лемма о рукопожатиях, 2021 URL: https://ru.wikipedia.org/wiki/Лемма_о_рукопожатиях

4. Васмут А.С. Моделирование в картографии с применением ЭВМ. - М.: Недра, 1983. - 200 с.

5. Кристофидес Н. Теория графов: Алгоритмический подход. - М.: Мир, 1978. - 432 с.

6. Смирнов C.B. Проектирование графических систем со сложной структурой данных. - Саарбрюкен: LAP Lambert Academic Publishing, 2011. - 176 c.

7. Samet H., Webber R. Storing a Collection of Polygons Using Quadtrees // ACM Transactions on Graphics, Vol. 4, No. 3, July 1985, P.P. 182-222.

8. Квадратомическое дерево, 2021 URL: https://studfile.net/preview/5375751/page:15/

9. Смирнов С.В. Построение изобразительных средств аналитической модели тематического слоя специализированной геоинформационной системы при помощи квадратомического дерева / Сборник статей Международной научно-практической конференции «Совершенствование методологии и организации научных исследований в целях развития общества» (Новосибирск, 29.12.2020 г.). Стерлитамак: АМИ, 2020. Ч. 2. С. $182-186$. 